\title{
Effet paradoxal du vieillissement sur des tâches de mémoire prospective time-based en situation de laboratoire et en milieu naturel : Rôle des fonctions exécutives
}

\author{
Barbara Azzopardi, ${ }^{1}$ Caroline Auffray, ${ }^{2}$ et Christophe Kermarrec ${ }^{2}$
}

\begin{abstract}
The current study investigates the specific role of three executive functions in the explanation of performance variability on laboratory and naturalistic time-based prospective memory tasks in aging. Ninety people aged 18 to 80 years performed three executive tasks assessing inhibition, flexibility and updating, one laboratory time-based task and one naturalistic time-based task. The results indicate that age has a negative impact on the laboratory time-based task and a positive impact on the naturalistic task. The mediation analysis shows that inhibition and flexibility operate as a mediator in the negative relationship between age and the laboratory time-based task. Additionally, the hierarchical regression analyses show that, after controlling for age, none of the executive functions evaluated is predictive of performance of the naturalistic time-based task. Overall, these results seem to suggest that different cognitive processes underlie the performance on naturalistic and on laboratory time-based tasks. Future studies should confirm these results.
\end{abstract}

\section{RÉSUMÉ}

Cette recherche étudie le rôle spécifique de trois fonctions exécutives dans l'explication des différences de performances à des tâches de mémoire prospective time-based conduites en laboratoire et en milieu naturel au cours du vieillissement. Quatre-vingt-dix adultes âgés de 18 à 80 ans ont effectué trois tâches évaluant l'inhibition, la flexibilité et la mise à jour, une tâche time-based de laboratoire et une tâche time-based naturelle. Les résultats ont montré que l'âge a un effet négatif sur la tâche time-based de laboratoire et un effet positif sur la tâche naturelle. L'inhibition et la flexibilité médiatisent totalement l'effet de l'âge sur la performance à la tâche de laboratoire. Par ailleurs, après contrôle des effets de l'âge, aucune des fonctions exécutives évaluées n'est prédictrice de la performance à la tâche naturelle. Ces résultats semblent suggérer que les performances à des tâches time-based de laboratoire et naturelles pourraient être sous-tendues par des processus cognitifs différents. Des études futures devraient permettre de confirmer ces résultats.

1 Centre de Recherche en Psychologie de la Connaissance, du Langage et de l'Émotion (PsyClé), Université Aix-Marseille, France

2 Centre de Recherches en Psychologie, Cognition, Communication (CRPCC), Université de Bretagne Occidentale, Brest, France Manuscript received: / manuscrit reçu : 09/06/15

Manuscript accepted: / manuscrit accepté : 10/04/16

Keywords: aging, executive functions, prospective memory, laboratory task, naturalistic task, time-based task

Mots clés : vieillissement, fonctions exécutives, mémoire prospective, tâche de laboratoire, tâche naturelle, tâche time-based La correspondance et les demandes de tirés à part doivent être adressées à : / Correspondence and requests for offprints should be sent to:

Barbara Azzopardi (centre PsyClé)

Aix-Marseille Université,

29 Avenue Robert Schuman,

13100 Aix-en-Provence, France

(barbara.azzopardi@gmail.com)

\section{Introduction}

La mémoire prospective renvoie à la capacité d'une personne à se souvenir d'effectuer une action qu'elle a prévu d'accomplir dans le futur. Cette capacité est sollicitée quotidiennement chez les personnes âgées qui sont, par exemple, fréquemment amenées à se souvenir de prendre un traitement médicamenteux à différents moments de la journée.

La mémoire prospective est généralement mesurée dans deux grandes catégories de situations. Les tâches 
de laboratoire sont des tâches informatisées qui s'inspirent le plus souvent du paradigme d'Einstein \& McDaniel (1990), dans lequel le participant est placé en situation de " double tâche " pour simuler la réalisation d'une tâche de mémoire prospective dans la vie quotidienne. La tâche secondaire, appelée « tâche en cours », consiste par exemple à mémoriser des mots, dénommer des visages célèbres ou répondre à des questions sur un texte. Parallèlement, le participant doit se souvenir d'effectuer une action (p. ex. appuyer sur une touche du clavier, délivrer un message) : (a) à une heure précise (p. ex., $10 \mathrm{~h}$ 30) ou en respectant une certaine régularité (p. ex., toutes les trois minutes) dans les tâches dites time-based; (b) lorsqu'un indice exogène lui est présenté (p. ex., un mot d'une catégorie supraordonnée), dans les tâches dites event-based. Excepté quelques études (p. ex., Rendell \& Thomson, 1999), les tâches naturelles sont le plus souvent réalisées sans contrôle de l'expérimentateur dans le milieu naturel du participant et sont intégrées à ses activités quotidiennes. À la différence des tâches de laboratoire, qui ne durent généralement que quelques minutes, les tâches naturelles s'étendent souvent sur plusieurs jours (Bailey, Henry, Rendell, Phillips, \& Kliegel, 2010). Bien que la distinction entre tâches time-based et event-based ait classiquement été appliquée aux tâches de laboratoire de mémoire prospective, et que les tâches naturelles utilisées dans les études empiriques soient généralement de type time-based, la distinction time-based vs event-based peut également s'appliquer aux tâches naturelles de mémoire prospective. Dans une tâche de type time-based, le participant doit, par exemple, se souvenir de poster une lettre à l'expérimentateur un jour précis ou d'appeler l'expérimentateur à une heure précise pendant plusieurs jours. Dans une tâche de type event-based, le participant doit, par exemple, se souvenir d'appeler l'expérimentateur dès qu'il verra à la télévision la carte de la météo durant les prévisions météorologiques du soir (Niedźwieńska \& Barzykowski, 2012). Dans la majorité des travaux portant sur la mémoire prospective, celle-ci est évaluée par des tâches de laboratoire, le plus souvent de type event-based ; les mécanismes sous-tendant les effets du vieillissement sur la performance aux tâches time-based, sont moins bien connus (Cona, Arcara, Tarantino, \& Bisiacchi, 2012). Cette recherche est donc centrée sur les mécanismes sous-tendant les effets du vieillissement sur des tâches time-based de laboratoire et conduites en milieu naturel.

La comparaison de personnes âgées et jeunes à des tâches time-based de mémoire prospective a conduit à deux résultats contradictoires regroupés sous le nom de " effet paradoxal de l'âge sur la mémoire prospective » (Henry, MacLeod, Phillips, \& Crawford, 2004; Phillips, Henry, \& Martin, 2008; Rendell \& Craik, 2000; Uttl, 2011). Le premier résultat est conforme à ce que l'on sait des effets du vieillissement sur le fonctionnement cognitif (e.g., Craik \& Salthouse, 2007), les personnes âgées étant en moyenne moins performantes dans les tâches de laboratoire que les personnes jeunes (Gonneaud et al., 2011; Henry et al., 2004; Martin, Kliegel, \& McDaniel, 2003; McFarland \& Glisky, 2009; Mioni \& Stablum, 2014; Phillips et al., 2008; Rendell \& Thomson, 1999). Ce résultat classique paraît être contredit par le constat d'une meilleure performance des personnes âgées, en comparaison aux plus jeunes, dans les tâches naturelles (Bailey et al., 2010; Niedźwieńska \& Barzykowski, 2012; Rendell \& Thomson, 1999; Schnitzspahn, Ihle, Henry, Rendell, \& Kliegel, 2011). L'approche généralement employée consiste en effet à comparer la performance moyenne de sujets âgés à celle de jeunes adultes. Si la comparaison entre groupes d'âge différent permet de repérer les tendances moyennes dans les changements liés à l'âge, les groupes d'âge sont implicitement considérés comme homogènes (Zeintl, Kliegel, \& Hofer, 2007). Un tel plan d'analyse, contrairement à une approche différentielle, ne peut donc tenir compte de l'accroissement lié à l'âge de la variabilité interindividuelle (Christensen et al., 1999; West, Murphy, Armilio, Craik, \& Stuss, 2002). Un autre intérêt de l'approche interindividuelle est qu'elle permet d'étudier l'évolution avec l'âge du déclin de la performance de mémoire prospective. Les quelques études ayant examiné la trajectoire du déclin lié à l'âge de la performance de mémoire prospective chez l'adulte ont conduit à des résultats contradictoires. En effet, certaines de ces études ont montré que le déclin lié à l'âge de la performance de mémoire prospective était linéaire (Huppert, Johnson, \& Nickson, 2000 ; Zeintl et al., 2007), tandis que d'autres ont mis en évidence une accélération de ce déclin chez les plus âgés (Kliegel, Mackinlay, \& Jäger, 2008; Salthouse, Berish, \& Siedlecki, 2004). Notons également que les résultats de ces études sont limités exclusivement aux tâches event-based. Les connaissances dans ce domaine apparaissent donc limitées. Une approche différentielle sur un échantillon d'une large étendue d'âge (Huppert et al., 2000 ; Zeintl et al., 2007) paraît alors à même de compléter utilement l'approche comparative jeunes vs âgés habituellement employée pour étudier les effets du vieillissement sur la performance aux tâches time-based naturelles et de laboratoire de mémoire prospective.

La mise en évidence d'effets différents de l'âge sur la performance aux tâches time-based de laboratoire et conduites en situation naturelle amène à s'interroger sur la nature des processus cognitifs sous-tendant la performance dans ces deux types de situations d'évaluation. L'une des caractéristiques principales des tâches time-based de mémoire prospective est 
qu'elles nécessitent l'initiation par le sujet du déclenchement du souvenir. Ces tâches dites à faible support environnemental sollicitent donc fortement des processus exécutifs (Gonneaud et al., 2011; Martin et al., 2003; McFarland \& Glisky, 2009; Mioni \& Stablum, 2014). Or, on sait que le déclin lié à l'âge de la performance mnésique est plus marqué lorsque les processus sollicités sont auto-initiés plutôt que soutenus par des indices contextuels externes (Craik, 1986). Plusieurs travaux ont ainsi montré que le déclin lié à l'âge des fonctions exécutives (généralement évaluées par un score composite) jouait un rôle dans l'explication des effets de l'âge sur la performance à des tâches time-based de laboratoire (Martin et al., 2003; McFarland \& Glisky, 2009).

Si le rôle spécifique de l'inhibition dans l'explication des effets de l'âge sur la performance aux tâches time-based de laboratoire a été solidement établi (e.g., Gonneaud et al., 2011; Mioni \& Stablum, 2014), le rôle des deux autres principales fonctions exécutives définies par Miyake (2000) reste mal connu. La mise à jour pourrait notamment être impliquée dans la planification et dans le maintien temporaire de l'intention en mémoire de travail (Mioni \& Stablum, 2014). Cependant, les données de la littérature apparaissent contradictoires. Certaines études n'ont pas mis en évidence de relation entre les capacités de mise à jour et la performance time-based de mémoire prospective chez la personne âgée (Gonneaud et al., 2011, voir aussi Schnitzspahn, Stahl, Zeintl, Kaller, \& Kliegel, 2013, pour des résultats similaires avec des tâches eventbased), tandis que d'autres ont montré que ces capacités jouaient un rôle important dans l'explication des différences liées à l'âge aux tâches time-based (Mioni \& Stablum, 2014).

Deux recherches ont étudié le rôle spécifique de la flexibilité dans la performance de mémoire prospective de personnes âgées. Les résultats de l'étude de Schnitzspahn et al. (2013), conduite auprès d'un large échantillon de personnes jeunes $(n=175)$ et âgées $(n=110)$, ont révélé que les différences de performances à des tâches évaluant la flexibilité permettaient d'expliquer les différences de performances liées à l'âge à des tâches event-based de mémoire prospective. Toutefois, les résultats d'une autre recherche (Gonneaud et al., 2011) n'ont pas mis en évidence de relation significative entre l'efficience de la flexibilité et la performance à des tâches event-based et time-based de mémoire prospective chez des personnes âgées. S'il existe plusieurs arguments en faveur de l'hypothèse selon laquelle le déclin lié à l'âge de la performance aux tâches de mémoire prospective pourrait être expliqué, au moins en partie, par une diminution liée à l'âge de l'efficience de la flexibilité, les résultats des quelques recherches empiriques ayant abordé cette question permettent encore difficilement de conclure.
Seule une étude a examiné les relations entre les fonctions exécutives et la performance aux tâches timebased naturelles au cours du vieillissement (Cuttler \& Graf, 2007). Les résultats de cette étude conduite auprès d'un échantillon de personnes âgées de 18 à 81 ans $n$ 'ont pas mis en évidence de relation entre la performance à une tâche naturelle time-based et la performance à des tâches évaluant les capacités d'inhibition et de flexibilité.

L'objectif de ce travail est d'étudier, sur un échantillon de personnes âgées de 18 à 80 ans, le rôle spécifique de trois principales fonctions exécutives (inhibition, flexibilité, mise à jour) dans la relation entre l'âge, considéré comme continu, et la performance à des tâches time-based de laboratoire et naturelle. Nous nous attendons, tout d'abord, conformément aux résultats rappelés précédemment, à observer une diminution liée à l'âge de la performance à une tâche time-based de laboratoire ainsi qu'à une amélioration avec l'âge de la performance à une tâche time-based naturelle. Nous chercherons à déterminer si la trajectoire des effets de l'âge sur la performance aux tâches de time-based est linéaire ou accélérée chez les plus âgés. Nous faisons également l'hypothèse que les fonctions d'inhibition, de flexibilité et de mise à jour devraient agir comme médiateurs dans la relation entre l'âge et la performance à la tâche time-based de laboratoire. Enfin, si contrairement à la tâche de laboratoire, la performance à la tâche naturelle n'est effectivement pas négativement affectée par l'âge, nous faisons l'hypothèse que les fonctions exécutives ne seront pas des prédicteurs de la performance à cette tâche.

\section{Méthode}

Participants

L'échantillon est constitué de 90 personnes âgées de 18 à 80 ans. Trente participants étaient des adultes jeunes âgés de 18 à 34 ans $\left(M_{\hat{a g g e}}=24,9\right.$ ans, E.T. $=4,46$, 19 femmes), trente étaient des adultes d'âge moyen âgés de 36 à 59 ans $\left(M_{\text {âge }}=46,9\right.$ ans, E.T. $=7,53$, 16 femmes), et trente étaient des adultes âgés de 62 à 80 ans $\left(M_{\hat{a} g e}=71,5\right.$ ans, E.T. $=5,35,22$ femmes $)$. Tous les participants vivaient à domicile de façon autonome, étaient français et avaient une audition et une vue normales ou corrigées. Parmi les participants âgés de 60 et plus, seuls ceux obtenant un score supérieur ou égal à 26 au MMSE (Mini Mental State Examination; Folstein, Folstein, \& McHugh, 1975) étaient inclus dans l'étude. Le score moyen au MMSE pour le groupe d'adultes âgés est de 28,67 (E.T. = 1,03). Le niveau d'études diffère significativement en fonction du groupe d'âge $(\mathrm{F}(2 ; 87)=4903 ; p<0,01)$. La différence entre le niveau d'études moyen du groupe d'adultes jeunes et celui du groupe d'âge moyen n'est pas significative (HSD Tukey, $p=.39$ ). Le niveau d'études 
des participants du groupe d'adultes âgés est plus faible que celui des adultes jeunes (HSD Tukey, $p<.01$ ) et d'âge moyen (HSD Tukey, $p<.05$ ). Les statistiques descriptives pour les variables démographiques et cognitives sont présentées dans le tableau 1.

\section{Matériel}

Tâche time-based de laboratoire. La tâche employée est adaptée de celle élaborée par Einstein, McDaniel, Richardson, Guynn, \& Cunfer (expérience 3, 1995). Les participants réalisent une épreuve informatisée de culture générale dans laquelle ils doivent juger si l'affirmation présentée à l'écran, en noir sur fond blanc, est vraie ou fausse. Afin de favoriser l'engagement des participants dans la réalisation de cette épreuve, un feedback visuel (smiley souriant ou mécontent) est fourni après chaque réponse. Parallèlement, les participants doivent se souvenir d'appuyer sur la barre d'espace toutes les deux minutes au lieu de répondre à la question affichée à l'écran. Ils disposent d'un chronomètre placé à un angle de $90^{\circ}$ par rapport à l'écran leur permettant de vérifier le temps écoulé (voir Gonneaud et al. 2011). L'épreuve comporte 76 questions qui apparaissent successivement à l'écran pendant une durée maximum de 10 secondes et dure au total 12 minutes 40 secondes. La précision à la tâche de mémoire prospective est donc mesurée à 6 occasions. Le participant dispose de cinq secondes après chaque temps cible (c.-à-d., 2 minutes, 4 minutes, 6 minutes, 8 minutes, 10 minutes et 12 minutes) pour fournir sa réponse prospective, au-delà de cette fenêtre temporelle, la réponse du participant n'est pas prise en compte et il obtient un score de zéro. Le nombre de vérifications du chronomètre avant chaque moment cible est mesuré. Nous avons également calculé un score de vérification stratégique (voir Voigt et al., 2014) qui correspond à la moyenne des différences, calculées pour les six occasions de mesures, entre le nombre de vérifications durant les 60 dernières secondes précédant le moment cible et le nombre de vérifications durant les 60 premières secondes précédant le moment cible.

Cette tâche naturelle time-based est adaptée de celle employée dans l'étude de Meacham et Singer (1977). Chaque participant recevait au début de l'étude un paquet de 4 enveloppes pré-timbrées avec pour consigne de poster une lettre le même jour de la semaine pendant quatre semaines. Il lui était également demandé de poster toute lettre oubliée dès la prise de conscience de l'oubli. On mesure le nombre de lettres postées le jour prévu (1 pour chaque lettre postée le jour prévu, 0 dans le cas contraire, soit un score maximal de 4 ).

\section{Mesure de flexibilité}

Nous avons utilisé le Trail Making Test (TMT) afin de mesurer la flexibilité. Dans la première partie du test (TMT-A), il faut relier le plus rapidement possible les nombres de 1 à 25 disposés de façon aléatoire sur une feuille. Dans la seconde partie du test (TMT-B), il faut relier le plus rapidement possible des lettres et des chiffres en alternant entre ceux-ci (1-A-2-B-3-C...). Nous avons calculé le score de différence qui correspond à la différence entre les temps de réalisation au TMT-B et au TMT-A (Arbuthnott \& Frank, 2000).

Tableau 1 : Moyennes et écarts-types (entre parenthèses) en fonction des groupes d'âge pour les variables démographiques et cognitives

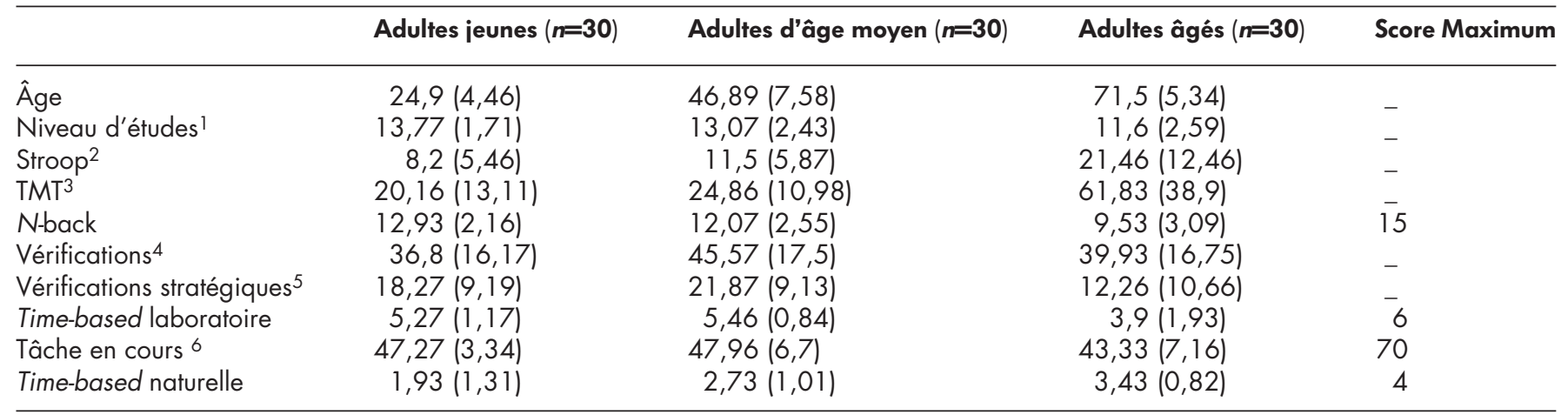

1 Le niveau d'études est donné en nombre d'années de scolarisation depuis le cours primaire.

2 Les scores présentés correspondent aux scores de différence (temps de réalisation de la planche interférence - temps de réalisation de la planche couleur).

3 Les scores correspondent aux scores de différences (TMT.B-TMT.A).

4 Nombre moyen de vérifications sur l'ensemble de l'épreuve.

5 Moyenne des différences entre le nombre de vérifications durant les 60 dernières secondes précédant le moment cible et le nombre de vérifications durant les 60 premières secondes précédant le moment cible pour les 6 occasions de mesure.

6 Score sur $\mathbf{7 0}$ au questionnaire réalisé durant la tâche time-based de laboratoire (les six questions correspondant aux 6 moments cibles n'ont pas été prises en compte). 
Ce score de différence constitue pour plusieurs auteurs un indicateur des capacités de flexibilité (Monsell, 2005; Sánchez-Cubillo et al., 2009).

\section{Mesure de l'inhibition}

Nous avons utilisé l'adaptation francophone du test du Stroop Victoria, (Bayard, Erkes, \& Moroni, 2009). Ce test est utilisé classiquement pour mesurer la résistance à l'interférence (McDowd \& Hoffman, 2008). Il comporte trois parties. Dans la première partie (planche couleur), on présente une planche comportant des points de couleurs différentes (Jaune, Vert, Rouge, Bleu) que le participant doit dénommer le plus rapidement possible sans faire d'erreur. Dans la seconde partie, on présente une planche comportant des mots (" mais », «pour », « donc », « quand ») écrits dans des couleurs différentes (Jaune, Vert, Rouge, Bleu), que le participant doit lire le plus rapidement possible sans faire $d^{\prime}$ erreur. Dans la dernière partie (planche interférente), on présente au participant une planche sur laquelle figurent des mots de couleurs (Jaune, Vert, Rouge, Bleu), écrits dans des couleurs différentes (Jaune, Vert, Rouge, Bleu). Le participant ne doit pas lire les mots mais dénommer le plus rapidement possible, sans faire d'erreur, la couleur de l'encre dans laquelle ils sont écrits. Pour chaque partie, le temps total de réalisation est recueilli. Afin de mesurer l'efficience de l'inhibition, nous avons ensuite calculé un score de différence obtenu en soustrayant le temps de réalisation de la planche couleur au temps de réalisation de la planche interférente.

\section{Mesure de la mise à jour}

Nous avons utilisé une tâche informatisée de n-back " mot », qui est classiquement utilisée pour évaluer les capacités de mise à jour en mémoire de travail. Dans cette tâche, des mots (noms communs de deux syllabes) sont successivement présentés à l'écran durant 1000 ms. Afin que la tâche soit adaptée à un échantillon de personnes âgées de 18 à 80 ans, nous avons utilisé une tâche de 2-back. Les participants ont donc pour consigne d'appuyer sur la barre d'espace chaque fois qu'un mot présenté à l'écran est identique à celui qui a été présenté deux essais auparavant. La tâche est composée de 46 essais parmi lesquels 15 étaient des essais cibles (i.e., 2-back), 6 étaient des essais leurres (c.à-d., 3 essais 1-back et 3 essais 3-back) et 25 étaient des essais non-cibles. La précision était donc mesurée à 15 occasions.

\section{Résultats}

Nous présentons dans cette section les résultats de modèles linéaires. Notons toutefois que la distribution des scores aux tâches de mémoire prospective n'étant pas normale, nous avons donc également appliqué des modèles de régression logistique pour données ordinales et nous sommes assurés que les résultats obtenus étaient systématiquement similaires à ceux présentés ici. Le tableau 1 présente les résultats descriptifs en fonction des trois groupes d'âges pour les variables démographiques et cognitives. Nous avons effectué des ANOVAs simples afin de comparer les scores sur chacune de ces variables en fonction des groupes d'âge. Les analyses ont mis en évidence un effet du groupe d'âge sur les scores au Stroop $(\mathrm{F}(2 ; 87)=14,78$; $p<.001)$. Les analyses post-hoc révèlent des différences significatives entre le groupe 1 (HSD Tukey, $p<.001$ ) et entre le groupe 2 et le groupe 3 (HSD Tukey, $p<.01$ ). Les scores au TMT diffèrent en fonction des groupes d'âge $(\mathrm{F}(2 ; 87)=23,97 ; p<.001)$. Seules les différences entre le groupe 1 et le groupe 3 (HDS Tukey, $p<.001$ ) et entre le groupe 2 et le groupe 3 (HSD Tukey, $p<.001$ ) sont significatives. Les scores de précision à la N-back diffèrent également en fonction du groupe d'âge $(\mathrm{F}(2 ; 85)=13475$; $p<.001)$. Il existe une différence significative entre le groupe 1 et le groupe 3 (HSD Tukey, $p<.001$ ) et entre le groupe 2 et le groupe $3(p<.01)$. Les scores à la tâche time-based de laboratoire sont différents en fonction du groupe d'âge $(\mathrm{F}(2 ; 87)=10,35 ; p<.001)$. Seules les différences de performances entre le groupe 1 et le groupe 3 (HSD de Tukey, $p<.01$ ) et entre le groupe 2 et le groupe 3 (HSD de Tukey, $p<.001$ ) sont statistiquement significatives. Le nombre moyen de vérifications n'est pas différent selon le groupe d'âge $(\mathrm{F}(2 ; 87)=2,59 ; p=.08)$. Néanmoins, les vérifications stratégiques diffèrent selon le groupe d'âge $(\mathrm{F}(2 ; 87)=4,67 ; p<.05)$. Seule la différence entre le groupe 1 et le groupe 3 est statistiquement significative (HDS de Tukey, $p<.01$ ). Les performances à la tâche en cours diffèrent en fonction du groupe d'âge $(\mathrm{F}(2 ; 87)=4,29 ; p<.05)$. Seule la différence de scores entre le groupe 2 et le groupe 3 est statistiquement significative (HDS Tukey, $p<.05$ ).

\section{Étude des relations entre l'âge et la performance aux tâches time-based}

Afin d'étudier les relations entre l'âge et la performance aux tâches de mémoire prospective (voir figure 1), nous avons comparé les résultats du modèle de régression linéaire simple et du modèle de régression avec effet quadratique de l'âge. L'ajustement du modèle a été estimé à partir du Bayesian Information Criterion (BIC), un critère d'ajustement qui tient compte à la fois de la vraisemblance et de la parcimonie du modèle. Cet indicateur est calculé pour chacun des modèles estimés, et nous comparons ensuite ces modèles en retenant celui dont le BIC est le plus petit.

Les analyses de régression linéaire simple ont mis en évidence un effet négatif de l'âge sur la performance à la tâche time-based de laboratoire $(\beta=-.410 ; p<.001$, $\mathrm{BIC}=330)$. Un second modèle de régression a mis en 


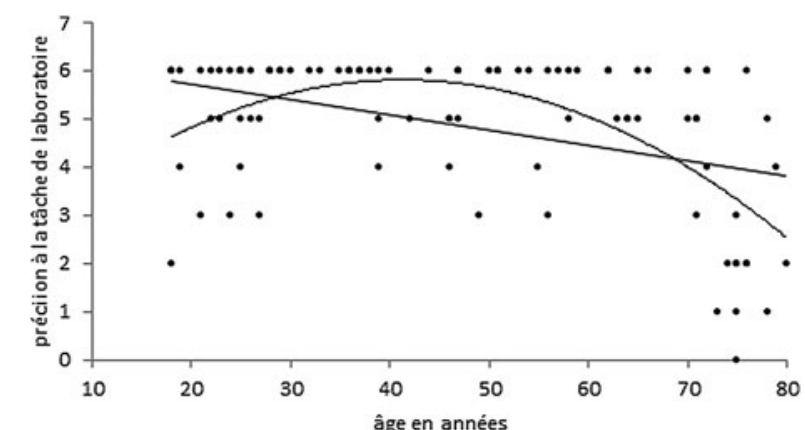

(a)

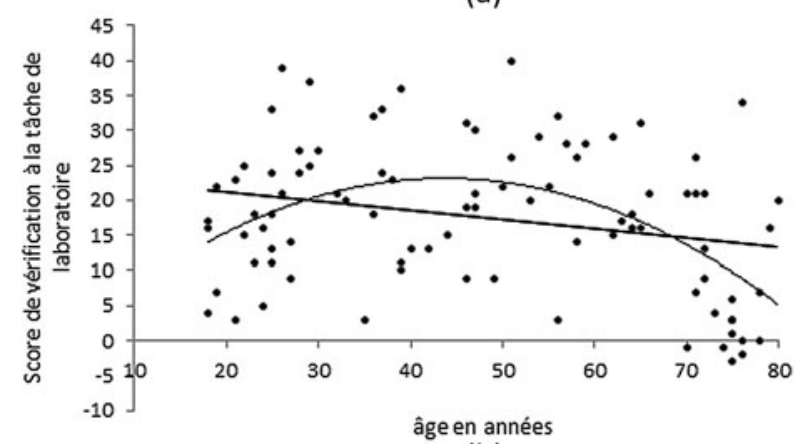

(b)

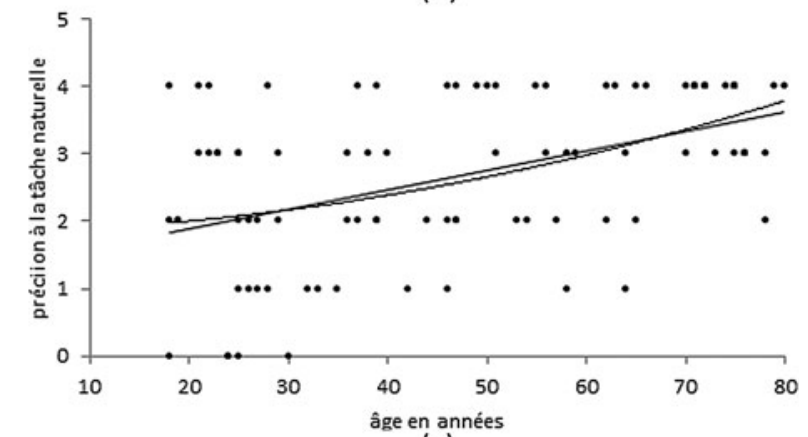

(c)

Figure 1: Nuages de points de la précision (a) et des scores de vérification (b) à la tâche time-based de laboratoire et de la précision à la tâche time-based naturelle (c) en fonction de l'âge. Les lignes droites représentent les effets linéaires, les lignes courbes les effets quadratiques

évidence un effet quadratique significatif de l'âge sur la performance à la tâche time-based de laboratoire (estimation : $-0,002, p<.001, \mathrm{BIC}=312$ ). La représentation graphique de ces effets apparaît sur la figure 1 (a). Les résultats des analyses de régression simple ont mis en évidence un effet négatif de l'âge sur les vérifications stratégiques $(\beta=-.251 ; p<.05, \mathrm{BIC}=684)$. Les résultats ont par ailleurs montré un effet quadratique significatif de l'âge sur les vérifications stratégiques (estimation : $-0,013, p<.001$, BIC $=672$, voir figure 1 (b)). Pour ce qui est des relations entre l'âge et la tâche des lettres (voir figure $1(\mathrm{c})$ ), l'analyse de régression simple a mis en évidence un effet positif linéaire de l'âge sur la tâche des lettres $(\beta=.476 ; p<.001$, BIC=280), l'effet quadratique n'étant pas significatif (estimation : 0.0001, $p=.49$, BIC = 284).
Étude des relations entre l'âge, la performance aux tâches time-based et les fonctions exécutives

Corrélations entre les différentes mesures et l'âge. Le tableau 2 présente les corrélations entre les différentes mesures et l'âge et les corrélations partielles entre les différentes mesures après contrôle des effets de l'âge. Plusieurs résultats doivent être soulignés. Tout d'abord la tâche de mémoire prospective de laboratoire est significativement corrélée avec toutes les mesures et l'âge. Après contrôle des effets de l'âge, cette mesure n'est plus corrélée de façon significative avec la tâche naturelle de mémoire prospective ni avec la mise à jour. La tâche naturelle est également significativement corrélée avec toutes les mesures ; cependant, après contrôle des effets de l'âge, aucune des corrélations n'est significative. Les trois mesures exécutives sont corrélées entre elles, mais après contrôle des effets de l'âge, la mise à jour n'est plus significativement corrélée à la flexibilité et à l'inhibition.

\section{Régressions hiérarchiques}

Des analyses de régression hiérarchiques ont été réalisées afin d'étudier la contribution de l'âge et des différentes fonctions exécutives dans l'explication de la variance des performances aux tâches de mémoire prospective après contrôle du niveau d'études. Dans un premier modèle, l'analyse a montré, après contrôle du niveau d'études, que l'âge expliquait environ 17 pour cent de la variance à la tâche time-based de laboratoire (Modèle $1: R^{2}=.176, \beta=-.420, F=9.31$; $p<.001)$. Nous avons entré dans un second modèle le niveau d'études, l'âge, l'inhibition, la flexibilité et la mise à jour. Ces trois variables permettent d'expliquer environ 34 pour cent de variance supplémentaire à la variance expliquée par l'âge à la tâche time-based de laboratoire (Modèle $2: R^{2}=.515, \beta=-.717, F=17.38$, $p<.001)$. L'examen détaillé des résultats du modèle a révélé que l'inhibition $(\beta=-.298 ; \beta<.05)$ et la flexibilité $(\beta=-.544 ; p<.01)$ apportaient une contribution significative à l'explication de la variance à la tâche time-based de laboratoire, à la différence de la mise à jour $(\beta=.035 ; p=.706)$. Les analyses de régression hiérarchique concernant la tâche timebased naturelle ont montré, qu'après contrôle du niveau d'études, l'âge permettait de prédire environ 22 pour cent de la variance (Modèle $1: R^{2}=.226$, $\beta=.476, F=12.769, p<.001)$. L'ajout de l'inhibition, de la flexibilité et de la mise à jour dans le modèle ne permet d'expliquer qu'environ 3 pour cent de variance supplémentaire (Modèle $1: R^{2}=.252, \beta=.502$, $F=5.529, p<.001)$ et aucune de ces variables ne contribue de façon significative à l'explication de la variance à la tâche time-based naturelle (inhibition : $\beta=.11, \mathrm{p}=.423$; flexibilité $: \beta=.066, \mathrm{p}=.636$; mise à jour : $\beta=-.145, p=.212$ ). 
Tableau 2 : Corrélations entre l'âge et les différentes variables cognitives (partie inférieure de la diagonale) et corrélations partielles entre les différentes variables cognitives après contrôle des effets de l'âge (partie supérieure de la diagonale)

\begin{tabular}{|c|c|c|c|c|c|c|c|c|}
\hline & 1 & 2 & 3 & 4 & 5 & 6 & 7 & 8 \\
\hline 1-âge & & - & & & & & & \\
\hline 2-Time-based Laboratoire & $-0,41$ * & & $0,45^{*}$ & $-0,72^{*}$ & $-0,08$ & $-0,44^{*}$ & $-0,61$ * & 0,14 \\
\hline 3-Vérifications & $-0,07$ & $0,38^{*}$ & & $0,57^{*}$ & $-0,03$ & -.16 & $-0,16$ & 0,04 \\
\hline 4-Vérifications stratégiques & $-0,25$ & $0,74^{*}$ & $0, \overline{5} 3^{*}$ & & $-0,02$ & -.21 & $-0,35$ & 0,01 \\
\hline 5-Time-based naturelle & $0,48 *$ & $-0,26$ & 0,01 & $-0, \overline{1} 4$ & & 0,10 & 0,01 & $-0,15$ \\
\hline 6-Stroop & $0,60 *$ & $-0,62 *$ & $-0,07$ & $-0,38 *$ & $-0,36^{*}$ & & $-0,41^{*}$ & $-0,26$ \\
\hline 7-TMT & $0,60 *$ & $-0,69 *$ & $-0,09$ & $0,42 *$ & 0,29 & $0,60 *$ & & $-0,11$ \\
\hline 8- n-back & $-0,51$ * & 0,32 & 0,01 & 0,13 & $-0,36^{*}$ & $0,46^{*}$ & $-0, \overline{3} 8 *$ & - \\
\hline
\end{tabular}

Corrélations significatives ${ }^{*} \mathrm{p}<.001$, après correction de Bonferroni.

\section{Analyses de médiation}

Des analyses de médiation ont été conduites afin d'étudier le rôle médiateur des fonctions exécutives dans la relation entre l'âge et la performance à la tâche time-based de laboratoire. La procédure suivie est celle définie par Baron et Kenny's (1986), qui suggère que quatre critères doivent être remplis afin de mettre en évidence un effet de médiation: 1) une relation significative entre la variable dépendante et le prédicteur (en l'occurrence entre l'âge et la performance à la tâche time-based) ; 2) une relation significative entre le prédicteur et les médiateurs (c.-à-d., entre l'âge, l'inhibition, la flexibilité et la mise à jour) ; 3) Une relation significative entre la variable dépendante et les médiateurs (c.-à-d., relation significative entre la performance à la tâche time-based, l'inhibition, la flexibilité et la mise à jour). 4) Une réduction significative de l'amplitude de la relation entre la variable dépendante et le prédicteur après introduction des médiateurs dans le modèle (c.-à-d., réduction significative de l'effet direct de l'âge après introduction des médiateurs retenus après les étapes 2) et 3).

Conformément au premier critère, les analyses de régression simple ont mis en évidence un effet négatif de l'âge sur la performance à la tâche time-based $(\beta=-.410 ; p<.001)$. En accord avec le second critère, nous avons étudié les relations entre l'âge et les médiateurs. Les résultats ont montré que l'âge avait un effet significatif sur toutes les mesures évaluant fonctions exécutives (inhibition : $\beta=.603 ; p<.001$; flexibilité : $\beta=.601 ; p<.001$; mise à jour précision : $\beta=-.502 ; p<.001$ ). Dans un troisième temps, nous avons vérifié si ces différentes variables étaient des prédicteurs de la performance à la tâche time-based après contrôle des effets de l'âge. L'analyse des résultats a révélé que l'inhibition $(\beta=-.259 ; p<.01)$ et la flexibilité $(\beta=-.505$; $p<.001)$ étaient des prédicteurs négatifs et significatifs de la performance à la tâche time-based, tandis que la précision à la tâche de mise à jour $(\beta=.002$; $p=.985)$ n'était pas un prédicteur significatif de la performance à la tâche time-based. Enfin, nous avons testé un modèle dans lequel l'inhibition et la flexibilité médiatisent les effets de l'âge sur la performance à la tâche time-based. Les résultats de ce modèle sont présentés sur la figure 2. Ils ont montré qu'après introduction des médiateurs dans le modèle, l'effet direct de l'âge sur la performance à la tâche timebased de laboratoire n'est plus significatif $(\beta=.100$; $p=.323)$. L'âge a un effet délétère sur les scores de différence au TMT $(\beta=.601 ; p<.001)$ et au Stroop $(\beta=.603$; $p=.001)$. Les score de différence au TMT et au Stroop sont des prédicteurs de la performance à la tâche time-based de laboratoire (respectivement : $\beta=-.581$; $p<.001 ; \beta=-.306 ; p<.01)$. En accord avec les recommandations de Preacher \& Hayes (2008), l'estimation de la significativité de ces effets de médiation a été réalisée avec la procédure de bootstrap. L'effet de médiation peut être considéré comme significatif quand la valeur de « $0 »$ n'est pas comprise dans l'intervalle de confiance à 95\%. Les analyses réalisées sur 5000 échantillons bootstrap ont confirmé que les effets indirects de l'âge sur la performance à la tâche time-based de laboratoire via les scores de différence au TMT et au Stroop étaient significatifs (IC à $95 \%$ respectifs : [-.042- -.012]; [-.025- -.002]).

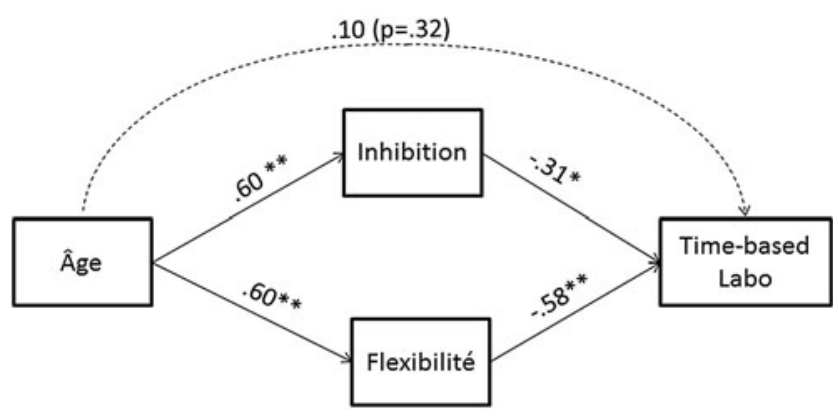

Figure 2: Résultats de l'analyse de médiation. Les nombres correspondent aux coefficients beta des analyses de régression. ${ }^{*} p<.01 ;{ }^{* *} p .<.001$. La flèche discontinue représente l'effet direct de l'âge sur la tâche time-based après introduction des médiateurs 


\section{Discussion}

L'objectif principal de cette recherche conduite dans une approche interindividuelle était d'étudier le rôle spécifique de trois fonctions exécutives dans la performance de tâches time-based de mémoire prospective de laboratoire et conduites en milieu naturel chez un échantillon de personnes âgées de 18 à 80 ans.

Conformément aux résultats de précédents travaux, nous avons tout d'abord mis en évidence un effet négatif de l'âge sur la précision et les vérifications stratégiques à la tâche time-based de laboratoire (Gonneaud et al., 2011; McFarland \& Glisky, 2009; Mioni \& Stablum, 2014). Nous avons également démontré que les effets de l'âge sur ces deux variables n'étaient pas linéaires. Ces résultats élargissent et renforcent ceux des quelques études ayant démontré que la trajectoire de développement de la performance aux tâches event-based de laboratoire était accélérée chez les personnes les plus âgées (Kliegel et al., 2008; Salthouse et al., 2004).

Les résultats ont par ailleurs mis en évidence que l'avancée en âge s'accompagnait d'une augmentation des performances à la tâche naturelle de mémoire prospective. Dans l'ensemble, ils confortent ceux des études comparatives ayant démontré que les jeunes obtenaient des performances en moyenne inférieures à celles des âgés dans ce type de situation (Bailey et al., 2010; Niedzwieñska \& Barzykowski, 2012; Rendell \& Thomson, 1999 ; Schnitzspahn et al., 2011). Toutefois cette étude est l'une des premières à notre connaissance à avoir confirmé cet effet paradoxal du vieillissement en ayant recours à une approche interindividuelle.

L'objectif central de cette recherche était d'étudier le rôle spécifique de l'inhibition, de la flexibilité et de la mise à jour dans la performance de tâches de mémoire prospective time-based de laboratoire et conduites en milieu naturel. Notons tout d'abord que l'analyse de nos résultats confirme le constat classique d'un déclin lié à l'âge de ces trois fonctions exécutives (pour revue, Braver \& West, 2008). Par ailleurs, l'analyse a montré qu'après contrôle des effets de l'âge, les performances à la tâche évaluant la mise à jour n'étaient plus corrélées à celles évaluant l'inhibition et la flexibilité et la corrélation entre ces deux dernières variables était affaiblie. Ces résultats apparaissent en accord avec ceux de travaux ayant mis en évidence une faible relation entre ces différentes fonctions exécutives (p. ex., Hull, Martin, Beier, Lane, \& Hamilton, 2008).

S'agissant ensuite des relations entre ces fonctions et la performance à la tâche time-based de laboratoire, nous avons montré qu'indépendamment des effets de l'âge, les différences individuelles de performance à la tâche de laboratoire pouvaient être prédites par des différences individuelles au niveau de l'inhibition et de la flexibilité. De surcroît, l'analyse de médiation a révélé que les effets de l'âge sur la performance à la tâche time-based de laboratoire étaient totalement médiatisés par les performances des participants aux tâches évaluant l'inhibition et la flexibilité. Le rôle de l'inhibition dans l'explication du déclin lié à l'âge aux tâches timebased de laboratoire a déjà été établi (Gonneaud et al., 2011; Mioni \& Stablum, 2014). Ce processus sensible aux effets de l'âge semble particulièrement sollicité dans les tâches de mémoire prospective time-based. Il est en effet notamment impliqué lorsque la personne qui est engagée dans une tâche de mémoire prospective doit identifier ou détecter le moment cible permettant le déclenchement du souvenir prospectif et interrompre son activité en cours (c.-à-d., inhiber la réalisation de cette activité) pour effectuer l'action prévue. Il n'est donc pas surprenant que les différences de performances à des tâches évaluant l'inhibition expliquent une part importante de la variance liée à l'âge aux tâches time-based de laboratoire.

Conformément aux résultats de précédents travaux portant sur la performance à des tâches event-based (Schnitzspahn et al., 2013), nos résultats suggèrent que la baisse d'efficacité de la flexibilité avec l'avancée en âge explique, au moins en partie, la diminution des performances time-based de mémoire prospective observée au cours du vieillissement. Les résultats de notre étude apportent donc des arguments empiriques supplémentaires à la théorie de PAM (The Preparatory Attentional and Memory processes : Smith, 2003; Smith \& Bayen, 2004) qui postule que la réussite à une tâche de mémoire prospective dépendrait de processus coûteux qui sous-tendraient notamment la capacité à alterner entre les processus nécessaires à la réalisation de l'activité en cours et ceux nécessaires à la détection du moment cible permettant la réalisation de l'action planifiée (voir aussi, Schnitzspahn et al., 2013).

Par ailleurs, en accord avec les résultats de l'étude de Gonneaud et al., (2011) et contrairement à ceux de Mioni et Stablum (2014), les résultats de notre étude ont montré que la mise à jour, telle qu'elle est évaluée, n'est pas impliquée dans la performance à la tâche time-based de laboratoire. Cette divergence dans les résultats pourrait être expliquée par des différences dans la construction des tâches time-based employées dans ces études. Dans l'étude de Mioni et Stablum, les participants avaient pour consigne d'appuyer sur une touche toutes les cinq minutes durant le visionnage d'un film. La fréquence des cibles prospectives était donc plus faible dans leur étude que dans la nôtre. Cette différence dans la construction des tâches pourrait être à l'origine de différences dans la nature des processus cognitifs sous-tendant leurs performances. Cette divergence de résultats pourrait également provenir d'une différence au niveau de l'étendue d'âge 
des participants constituant les échantillons expérimentaux. Les participants de la présente étude et ceux de l'étude de Gonneaud et al., (2011) étaient âgés de 18 à plus de 80 ans, tandis que l'étude Mioni et Stablum (2014) n'incluait pas de participants d'âge moyen. Or, il est possible que les capacités de mise à jour ne soient impliquées dans la performance aux tâches time-based qu'à certains moments du développement. Il a par exemple été démontré que la mise à jour avait un impact plus important dans les performances d'adolescents de 14 ans que chez des enfants âgés de moins de 10 ans (Voigt et al., 2014).

Enfin, le dernier résultat de cette étude concerne l'absence de relation entre la performance aux tâches évaluant les fonctions exécutives et la tâche naturelle de mémoire prospective. Les analyses corrélationnelles ont dans un premier temps montré une relation entre ces tâches, toutefois l'analyse des corrélations partielles a révélé que cette relation était due à la relation entre l'âge et les performances à la tâche naturelle. Nous avons effectivement montré qu'après contrôle des effets de l'âge, les relations entre les performances aux tâches évaluant l'inhibition, la flexibilité et la mise à jour d'une part, et les performances à la tâche naturelle de mémoire prospective, d'autre part, n'étaient plus significatives.

Plusieurs hypothèses ont été avancées pour expliquer la supériorité des personnes âgées aux tâches naturelles de mémoire prospective telles qu'un cadre de vie plus routinier et plus structuré (Henry et al., 2004), un niveau plus élevé de "motivation » (Aberle, Rendell, Rose, McDaniel, \& Kliegel, 2010; Niedźwieńska \& Barzykowski, 2012; Schnitzspahn et al., 2011) ou encore l'utilisation plus fréquente d'aides mnésiques externes et de stratégies de compensation (Masumoto, Nishimura, Tabuchi, \& Fujita, 2011). Les résultats de cette étude suggèrent que l'effet paradoxal de l'âge sur la mémoire prospective peut également être expliqué par des différences au niveau des processus cognitifs sous-tendant la performance aux tâches de laboratoire et aux tâches naturelles. Ce constat, ajouté à l'absence de corrélation entre les tâches naturelles et de laboratoire, interroge leur validité en tant que mesures d'un seul et même construit psychologique reflétant l'efficience de la mémoire prospective. Les travaux dans ce domaine doivent se poursuivre afin de déterminer de façon plus précise si les tâches de mémoire prospective conduites en situation de laboratoire et en milieu naturel évaluent un même construit cognitif.

Bien que les résultats de cette étude contribuent à l'amélioration des connaissances dans le domaine du vieillissement de la mémoire prospective time-based, nous devons néanmoins signaler certaines limites méthodologiques. La première limite de cette étude concerne la faible étendue des scores de nos mesures de mémoire prospective. En effet, une faible étendue peut réduire la variabilité des scores et engendrer des effets plafond. Ce problème est récurrent dans les études portant sur la mémoire prospective et peut avoir comme conséquence de réduire artificiellement les effets de l'âge (Uttl, 2011). Cependant, la mise en évidence dans cette étude d'un effet négatif et significatif de l'âge sur la tâche de laboratoire et d'un effet positif et significatif de l'âge sur la tâche naturelle suggère que nos mesures avaient suffisamment de variance pour différencier les participants. Il serait intéressant de reproduire une étude de ce type en veillant à utiliser des mesures de mémoire prospective plus sensibles.

Nous devons également souligner que les processus cognitifs étudiés n'ont été mesurés que par une seule tâche. Des travaux futurs ayant recours à une procédure d'opérationnalisation multiple permettraient de renforcer les résultats de cette étude. De plus, il serait intéressant de reproduire une étude de ce type sur un échantillon plus large permettant de réaliser des analyses de médiation multigroupe afin de déterminer si la nature des processus sous-tendant la performance aux tâches time-based diffère en fonction des périodes développementales. Il conviendrait également d'étudier le rôle d'autres processus cognitifs telle la vitesse de traitement dans l'explication des effets paradoxaux de l'âge sur la mémoire prospective.

Enfin, les résultats de cette étude doivent être nuancés dans la mesure où les deux tâches de mémoire prospective utilisées présentent des différences qui pourraient être contrôlées dans des études futures. Il serait notamment intéressant de reproduire une étude de ce type en veillant à créer des situations de laboratoire et naturelles dans lesquelles les délais de rétention seraient similaires et où l'utilisation d'aides externes serait contrôlée. Il est en effet possible que l'effet positif de l'âge sur les performances à la tâche naturelle de mémoire prospective puisse être expliqué par l'utilisation plus efficace de stratégies mnésiques compensatoires qui, en retour, modifierait la nature des processus cognitifs soustendant la tâche.

En résumé, cette étude est la première à notre connaissance à avoir examiné le rôle spécifique de l'inhibition, de la flexibilité et de la mise à jour, dans la performance de tâches time-based de laboratoire et conduites en milieu naturel, dans une approche interindividuelle auprès d'un échantillon présentant une large étendue d'âge. Les résultats ont permis de confirmer l'effet paradoxal du vieillissement sur la mémoire prospective. Ils ont par ailleurs montré que si l'effet positif de l'âge sur la performance à la tâche naturelle était linéaire, l'effet négatif de l'âge sur la performance à la tâche de 
laboratoire était quant à lui accéléré chez les plus âgés. Enfin, nous avons montré que l'inhibition et la flexibilité jouaient un rôle important dans l'explication des effets de l'âge sur la tâche de laboratoire tandis qu'aucune des fonctions exécutives évaluées dans cette étude n'était prédictrice de la performance à la tâche naturelle. Les résultats de cette étude suggèrent donc que les tâches time-based de laboratoire et conduites en milieu naturel sont différemment affectées par l'âge et sous-tendues par des processus cognitifs distincts. Enfin, des recherches futures semblent nécessaires afin de confirmer ces résultats et de déterminer si ces deux types de tâches évaluent bien un seul et même construit cognitif reflétant l'efficience de la mémoire prospective.

\section{Références}

Aberle, I., Rendell, P. G., Rose, N. S., McDaniel, M. A., \& Kliegel, M. (2010). The age prospective memory paradox: Young adults may not give their best outside of the lab. Developmental Psychology, 46(6), 1444-1453.

Arbuthnott, K. \& Frank, J. (2000). Trail making test, part B as a measure of executive control: validation using a setswitching paradigm. Journal of Clinical and Experimental Neuropsychology (Neuropsychology, Development and Cognition: Section A), 22(4), 518-528.

Bailey, P. E., Henry, J. D., Rendell, P. G., Phillips, L. H., \& Kliegel, M. (2010). Dismantling the "age-prospective memory paradox": The classic laboratory paradigm simulated in a naturalistic setting. The Quarterly Journal of Experimental Psychology, 63(4), 646-652.

Baron, R. M. \& Kenny, D. A. (1986). The moderator-mediator variable distinction in social psychological research: Conceptual, strategic, and statistical considerations. Journal of Personality and Social Psychology, 51(6), 1173-1182.

Bayard, S., Erkes, J., et Moroni, C. (2009). Test du Stroop VictoriaAdaptation francophone : Matériel, consignes, procédure de cotation et données normatives. Retrieved from http:/ / nca.recherche.univ-lille3.fr/uploads/File/ f-SV\%20_Bayard\%20Erkes\%20Moroni\%20-\%20nov09_ \%20Manuel.pdf.

Braver, T. S. \& West, R. (2008). Working memory, executive control, and aging. In F. I. M. Craik \& T. A. Salthouse (Eds.), The handbook of aging and cognition. (3rd ed., pp. 311-372). New York, NY: Psychology Press.

Christensen, H., Mackinnon, A. J., Korten, A. E., Jorm, A. F., Henderson, A. S., Jacomb, P., et al. (1999). An analysis of diversity in the cognitive performance of elderly community dwellers: Individual differences in change scores as a function of age. Psychology and Aging, 14(3), 365-379.

Cona, G., Arcara, G., Tarantino, V., \& Bisiacchi, P. S. (2012). Age-related differences in the neural correlates of remembering time-based intentions. Neuropsychologia, 50(11), 2692-2704.
Craik, F. I. M. \& Salthouse, T. A. (2007). The handbook of aging and cognition (3rd ed.). New York, NY: Psychology Press.

Craik, F. I. M. (1986). Human memory and cognitive capabilities. Mechanisms and performances. In F. Klix \& H. Hagendorf (Eds.), A functional account of age differences in memory (pp. 395-422). Amsterdam: Elsevier Science Publishers.

Cuttler, C. \& Graf, P. (2007). Personality predicts prospective memory task performance: An adult lifespan study. Scandinavian Journal of Psychology, 48(3), 215-231.

Einstein, G. O. \& McDaniel, M. A. (1990). Normal aging and prospective memory. Journal of Experimental Psychology: Learning, Memory, and Cognition, 16(4), 717-726.

Einstein, G. O., McDaniel, M. A., Richardson, S. L., Guynn, M. J., \& Cunfer, A. R. (1995). Aging and prospective memory: Examining the influences of self-initiated retrieval processes. Journal of Experimental Psychology: Learning, Memory, and Cognition, 21(4), 996-1007.

Folstein, M. F., Folstein, S. E., \& McHugh, P. R. (1975). "Mini-mental state." A practical method for grading the cognitive state of patients for the clinician. Journal of psychiatric research, 12(3), 189-198.

Gonneaud, J., Kalpouzos, G., Bon, L., Viader, F., Eustache, F., \& Desgranges, B. (2011). Distinct and shared cognitive functions mediate event- and time-based prospective memory impairment in normal ageing. Memory, 19(4), 360-377.

Henry, J. D., MacLeod, M. S., Phillips, L. H., \& Crawford, J. R. (2004). A meta-analytic review of prospective memory and aging. Psychology and Aging, 19(1), 27-39.

Hull, R., Martin, R. C., Beier, M. E., Lane, D., \& Hamilton, A. C. (2008). Executive function in older adults: A structural equation modeling approach. Neuropsychology, 22(4), 508-522.

Huppert, F. A., Johnson, T., \& Nickson, J. (2000). High prevalence of prospective memory impairment in the elderly and in early-stage dementia: Findings from a population-based study. Applied Cognitive Psychology, 14(7), S63-S81.

Kliegel, M., Mackinlay, R., \& Jäger, T. (2008). Complex prospective memory: Development across the lifespan and the role of task interruption. Developmental Psychology, $44(2), 612-617$.

Martin, M., Kliegel, M., \& McDaniel, M. A. (2003). The involvement of executive functions in prospective memory performance of adults. International Journal of Psychology, 38(4), 195-206.

Masumoto, K., Nishimura, C., Tabuchi, M., \& Fujita, A. (2011). What factors influence prospective memory for elderly people in a naturalistic setting?: Naturalistic study of prospective memory. Japanese Psychological Research, 53(1), 30-41. 
McDowd, J. M. \& Hoffman, L. (2008). Challenges in attention. Measures, methods, and applications. In S. M. Hofer \& D. F. Alwin (Eds.), Handbook of cognitive aging: Interdisciplinary perspectives (pp. 122-133). Los Angeles, CA: Sage Publications.

McFarland, C. P. \& Glisky, E. L. (2009). Frontal lobe involvement in a task of time-based prospective memory. Neuropsychologia, 47(7), 1660-1669.

Meacham, J. A. \& Singer, J. (1977). Incentive effects in prospective remembering. The Journal of Psychology, 97(2), 191-197.

Mioni, G. \& Stablum, F. (2014). Monitoring behaviour in a time-based prospective memory task: The involvement of executive functions and time perception. Memory, 22(5), 536-552.

Miyake, A. (2000). The unity and diversity of executive functions and their contributions to complex "frontal lobe" tasks: A latent variable analysis. Cognitive Psychology, 41(1), 49-100.

Monsell, S. (2005). The chronometrics of task-set control. In J. Duncan, P. McLeod, \& L. Phillips (Eds.), Measuring the mind: Speed, control, and age (1st ed., pp. 161-190). New York, NY: Oxford University Press.

Niedźwieńska, A. \& Barzykowski, K. (2012). The age prospective memory paradox within the same sample in time-based and event-based tasks. Aging, Neuropsychology, and Cognition, 19(1-2), 58-83.

Phillips, L. H., Henry, J. D., \& Martin, M. (2008). Adult aging and prospective memory: The importance of ecological validity. In M. Kliegel, M. A. McDaniel, \& G. O. Einstein (Eds.), Prospective memory: Cognitive, neuroscience, developmental, and applied perspectives (pp. 161-185). New York, NY: Lawrence Erlbaum Associates.

Preacher, K. J. \& Hayes, A. F. (2008). Asymptotic and resampling strategies for assessing and comparing indirect effects in multiple mediator models. Behavior Research Methods, 40(3), 879-891.

Rendell, P. G. \& Craik, F. I. M. (2000). Virtual week and actual week: Age-related differences in prospective memory. Applied Cognitive Psychology, 14(7), S43-S62.

Rendell, P. G. \& Thomson, D. M. (1999). Aging and prospective memory: Differences between naturalistic and laboratory tasks. The Journals of Gerontology Series B: Psychological Sciences and Social Sciences, 54B(4), P256-P269.
Salthouse, T. A., Berish, D. E., \& Siedlecki, K. L. (2004). Construct validity and age sensitivity of prospective memory. Memory \& Cognition, 32(7), 1133-1148.

Sánchez-Cubillo, I., Periáñez, J. A., Adrover-Roig, D., Rodríguez-Sánchez, J. M., Ríos-Lago, M., Tirapu, J., et al. (2009). Construct validity of the Trail Making Test: Role of task-switching, working memory, inhibition/interference control, and visuomotor abilities. Journal of the International Neuropsychological Society, 15(03), 438.

Schnitzspahn, K. M., Ihle, A., Henry, J. D., Rendell, P. G., \& Kliegel, M. (2011). The age-prospective memory-paradox: An exploration of possible mechanisms. International Psychogeriatrics, 23(04), 583-592.

Schnitzspahn, K. M., Stahl, C., Zeintl, M., Kaller, C. P., \& Kliegel, M. (2013). The role of shifting, updating, and inhibition in prospective memory performance in young and older adults. Developmental Psychology, 49(8), 1544-1553.

Smith, R. E. (2003). The cost of remembering to remember in event-based prospective memory: Investigating the capacity demands of delayed intention performance. Journal of Experimental Psychology: Learning, Memory, and Cognition, 29(3), 347-361.

Smith, R. E. \& Bayen, U. J. (2004). A multinomial model of event-based prospective memory. Journal of Experimental Psychology: Learning, Memory, and Cognition, 30(4), 756-777.

Uttl, B. (2011). Transparent meta-analysis: Does aging spare prospective memory with focal vs. non-focal cues? PLoS ONE, 6(2), e16618.

Voigt, B., Mahy, C. E. V., Ellis, J., Schnitzspahn, K., Krause, I., Altgassen, M., et al. (2014). The development of timebased prospective memory in childhood: The role of working memory updating. Developmental Psychology, 50(10), 2393-2404.

West, R., Murphy, K. J., Armilio, M. L., Craik, F. I. M., \& Stuss, D. T. (2002). Lapses of intention and performance variability reveal age-related increases in fluctuations of executive control. Brain and Cognition, 49(3), 402-419.

Zeintl, M., Kliegel, M. \& Hofer, S. M. (2007). The role of processing resources in age-related prospective and retrospective memory within old age. Psychology and Aging, $22(4), 826-834$. 